\title{
A Fuzzy TOPSIS with Affinity Weight for Big Data Projects: Managing Cloud Solution Problems
}

\author{
Nurnadiah Zamri, Wan Suryani Wan Awang
}

\begin{abstract}
This study underlines a fuzzy decision making method for determining weights of criteria that constitute for solving cloud solution problems in managing big data projects. The weight determination of cloud for big data projects is crucial since many uncertain and vagueness criteria that need to be considered concurrently. Furthermore, these criteria involve network performance, schedule and traffic management of cloud solutions problem. In response to these challenges, the affinity set applies to Fuzzy TOPSIS (FTOPSIS) method to propose timedependent weights of three criteria for managing big data projects. A major advantage of the affinity weights is that it incorporates performance-traffic management relationships between all criteria. This affinity weight with FTOPSIS method helps to solve the cloud solution problems. This paper also includes the same examples with different methods to compare and validate the proposed method. The proposed seven-step of affinity weight with FTOPSIS method finally managed to solve the cloud solution problem and the result was beautifully consistent with the other two methods.
\end{abstract}

Index Terms - Affinity weight, big data, cloud, FTOPSIS.

\section{INTRODUCTION}

Decision making is the way that chooses between two or more choices. The choices can be things, action, thought and etc. It can be a process to a particular selection when surrounded by a number of factors, variables, alternatives, criteria, etc. [1]. One of the most well-known methods to select the best decision is Multi Criteria Decision Making (MCDM). MCDM is known as one of the appropriate methods to make a sensible, organize decisions [2]. The best decision is forming based on selection of the alternative towards respective attributes by experienced experts [3]. MCDM is useful in all situations and can be adopted in real life decisions. However, MCDM itself unsuitable to solve this complexity, due to the real world cases that involve lots of difficulty and ambiguity [4]. To date, several MCDM methods have been introduced so far. Analytic Hierarchical Process (AHP) [5], Data Envelopment Analysis (DEA) [6], [7], Analytic Network Process (ANP) [8], [9], Technique for Order Performance by Similarity to Ideal Solution (TOPSIS) [10], [11], and many more. To date, the main famous method among MCDM is TOPSIS.

TOPSIS is chosen throughout this paper due to its consistency and simple in computation process [12]. Besides, famously known as an easy to understood and less

Revised Manuscript Received on July 10, 2019.

Nurnadiah Zamri, Faculty of Informatics and Computing, University Sultan Zainal Abidin, Besut Campus, 22200 Besut, Terengganu, Malaysia.

Wan Suryani Wan Awang, Faculty of Informatics and Computing, University Sultan Zainal Abidin, Besut Campus, 22200 Besut, Terengganu, Malaysia. computational method [13]. Fuzzy TOPSIS (FTOPSIS) was introduced as an extension for the proposed of Fuzzy Set. FTOPSIS proposed by [14] is believed a better method compared to previous TOPSIS in term of undefined the ambiguous linguistic judgment. Since that, FTOPSIS has been used by many researchers in many fields. For example, in [15] proposed FTOPSIS towards the evaluation of sustainable acid rain control options. In [16] proposed an ANP with FTOPSIS to choose the optimal tourism site based coastal zone management. In [17] developed an ordered fuzzy numbers based FTOPSIS method for application on the manufacturer. In [18] investigated Tehran stock exchange using the extended FTOPSIS. Overall, FTOPSIS method can be applied in many areas including biology, environment, mathematics, financial and many more. Besides all these areas, computer science areas specifically big data is also one of the famous areas in FTOPSIS [19].

Big data can be interpreted as a massive flood of exabytes and beyond data. Big data has the ability to gather many data, handle, process, envisage in a technology capability [20]. Manually and traditional process is difficult to handle huge and high amount of big data [21]. Thus, need a method such as MCDM to handle this data. In [22] studied on city logistics planning sustainability using affinity diagram, AHP and FTOPSIS method. In [23] integrated procurement management of big data analyst using fuzzy VIKOR technique. In [24] selected an appropriate cloud solution problem using the combination of FTOPSIS with an intuitionistic fuzzy set method. However, the previous studies neglected the performance, schedule, traffic management in a network of cloud solutions problems. Besides, it is sometimes can cause a complex problem since many uncertain and vagueness criteria that need to be considered concurrently. Thus, the main objective of this study is to initiate the FTOPSIS with affinity weight for managing big data projects to solve cloud solution problems. The new method reacts to the time and inter-dependent of weights from the experts' decisions.

The rest of this study can be traced back as; Section II explains on the background of Fuzzy Sets (FSs), Triangular Fuzzy Number (TFN), and affinity set. Section III focusses on the development of a new assessment model that consists 


\section{A FUZZY TOPSIS WITH AFFINITY WEIGHT FOR BIG DATA PROJECTS: MANAGING CLOUD SOLUTION PROBLEMS}

of FTOPSIS with affinity weight. Then, a numerical example calculates in Section IV with the demonstration of the viability and relevancy of the projected method. Section $\mathrm{V}$ shows the evaluation between the projected and existing example. Section VI concludes.

\section{PRELIMINARIES}

Definitions that attached to the FTOPSIS and affinity weight present in this section.

\section{A. Definition 2.1 Fuzzy Sets [25]}

Zadeh (the person who introduced Fuzzy Sets (FSs) [25] discussed that a FS can be interpreted as a group of objects that consider grades of membership. The idea of FSs are generalized by classical sets.

Let $H$ be a universe of sets, where ${ }_{\mathrm{A}}(\mathrm{h})$ is a membership function. Each element $h$ in $H$ is a fuzzy number from 0 till 1. Thus, the formula for FSs is as in (1):

$$
\tilde{\mathrm{A}}=\left\{\left(\mathrm{h}, \mu_{\mathrm{A}}(\mathrm{h})\right) \mid \mathrm{h} \in \mathrm{H}\right\}
$$

\section{B. Definition 2.2 Triangular Fuzzy Number [26]}

Next, in [25] expended FSs into a triangular fuzzy number (TFN). TFN consists of $\bar{A}$ where $\bar{A}$ can be represented as $\bar{A}=(1, \mathrm{~m}, \mathrm{u})$. Thus, the membership function is given by

$$
A^{\prime}(h)=\left\{\begin{array}{l}
h 1 / m \quad 1,1 \text { h m } \\
u \text { h/u m, m h u } \\
0, \text { otherwise }
\end{array}\right.
$$

\section{Definition 2.3 Affinity Sets [27]}

The meaning of affinity itself can be delineated as a similarity. Affinity involves a relationship, linkage between a group of people or elements. The full definition of affinity is interpreted as follows.

Let $e$ and $A=$ a subject and an affinity set and $I=$ a subset of the time axis $[0,+\infty)$. Therefore, the function of affinity between $e$ and $\mathrm{A}$ is denoted as,

$$
M_{A}^{e}(t): I \rightarrow[0,1]
$$

where $t \rightarrow M_{A}^{e}(t)$

which $M_{A}^{e}(t)$ is the degree of affinity between the subject e and the affinity set A at time $t$. Affinity function is a fuzzy set between $[0,1]$.

\section{ASSESSMENT MODEL}

This section presents the six steps of the assessment model where FTOPSIS is integrated with affinity set to become FTOPSIS with affinity weight. The overview of the proposed step-by-step FTOPSIS with affinity weight steps is summarized as follows.

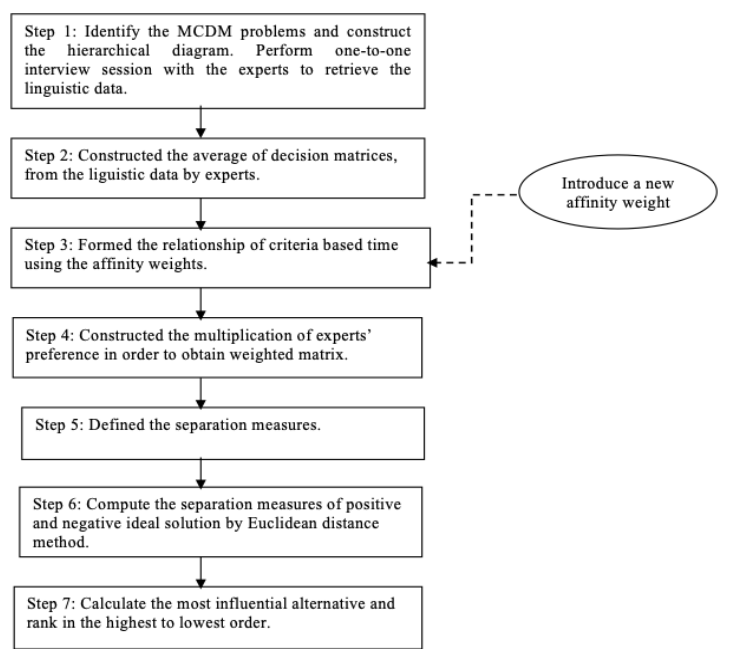

Fig. 1: A framework of the proposed FTOPSIS with affinity weights

The further explanations of the proposed seven steps of FTOPSIS with affinity weights are described as follows.

\section{A. Step 1. Linguistic Evaluation and Construct Decision Matrix}

Basically, linguistic scales are generated suited based on the decision problems. Here, cloud solution problems are our main target to solve. Therefore, the linguistic scales are scaled from Very Very Bad' to 'Extremely Good' and seen suited with the problems. Table 1 lists all the linguistic evaluation based on fuzzy sets. This linguistic evaluation is used by all experts to classify each alternative rating based factors.

Table 1: Linguistic evaluation of fuzzy sets

\begin{tabular}{|c|c|}
\hline Linguistic Evaluation & Scale \\
\hline (VVB) Very Very Bad & $(0,1,2)$ \\
\hline (VB) Very Bad & $(1,2,3)$ \\
\hline (B) Bad & $(2,3,4)$ \\
\hline (MB) Medium Bad & $(3,4,5)$ \\
\hline (F) Fair & $(4,5,6)$ \\
\hline (MG) Medium Good & $(5,6,7)$ \\
\hline (G) Good & $(6,7,8)$ \\
\hline (VG) Very Good & $(7,8,9)$ \\
\hline (VVG) Very Very Good & $(8,9,10)$ \\
\hline (EG) Extremely Good & $(9,10,1)$ \\
\hline
\end{tabular}

The construction of decision matrices in (4) is made using Table 1.

$$
\begin{aligned}
& \begin{array}{llll}
\mathrm{S}_{1} & \mathrm{~S}_{2} & \cdots & \mathrm{S}_{\mathrm{n}}
\end{array} \\
& \mathrm{E}=\left[\begin{array}{c}
\mathrm{B}_{1} \\
\mathrm{~B}_{2} \\
\vdots \\
\mathrm{B}_{\mathrm{M}}
\end{array}\left[\begin{array}{cccc}
\mathrm{g}_{11} & \mathrm{~g}_{12} & \cdots & \mathrm{g}_{1 \mathrm{n}} \\
\mathrm{g}_{21} & \mathrm{~g}_{22} & \cdots & \mathrm{g}_{2 \mathrm{n}} \\
\vdots & \vdots & \vdots & \vdots \\
\mathrm{g}_{\mathrm{m} 1} & \mathrm{~g}_{\mathrm{m} 2} & \cdots & \mathrm{g}_{\mathrm{mn}}
\end{array}\right]\right.
\end{aligned}
$$


where $\mathrm{S}_{1} \mathrm{~S}_{2} \cdots \mathrm{S}_{\mathrm{n}}$ are criteria, $\mathrm{B}_{1}, \mathrm{~B}_{2}, \ldots, \mathrm{B}_{\mathrm{m}}$ are alternatives and $\mathrm{g}_{\mathrm{ij}}$ is the rating of alternative.

\section{B. Step 2. Normalize the Linguistic Evaluation}

The normalized value $\left\{m_{i j}\right\}$ is defined as

$$
m_{i j}=\frac{\mathrm{g}_{\mathrm{ij}}}{\sqrt{\int_{\mathrm{j}=1}^{\mathrm{J}} \mathrm{g}_{\mathrm{ij}}^{2}}}, j=1, \ldots, J ; i=1, \ldots, n .
$$

\section{Step 3. Construct the Weighting Using Affinity Weight}

The weights using the affinity weights are constructed as follows:

$$
\begin{gathered}
\mathrm{w}_{\mathrm{r}_{\mathrm{ij}}}^{\mathrm{C}}(\mathrm{t}): \mathrm{I} \rightarrow[0,1] \\
t \rightarrow w_{r_{i j}}^{C}(t)
\end{gathered}
$$

where $\mathrm{w}_{\mathrm{r}_{\mathrm{ij}}}^{\mathrm{e}}(\mathrm{t})=$ the degree of affinity weight between the criteria $\mathrm{S}$ and the alternative $\mathrm{B}$ at time $t$.

Affinity function is a fuzzy set between [0,1]. Table 2 is needed to construct the weighting using affinity weight as in

Table 2: Linguistic evaluation of affinity weights

\begin{tabular}{|c|c|}
\hline Linguistic Evaluation & Affinity Weights \\
\hline (VW) Very Weak & $(0,1,2)$ \\
\hline (W) Weak & $(2,3,4)$ \\
\hline (M) Medium & $(4,5,6)$ \\
\hline (S) Strong & $(6,7,8)$ \\
\hline (VS) Very Strong & $(8,9,10)$ \\
\hline
\end{tabular}

\section{Step 4. Form the Decision Matrix's Weighted}

The $p_{i j}$ refers to the weighted normalized value as:

$\mathrm{p}_{\mathrm{ij}}=w_{i j} \times \mathrm{m}_{\mathrm{ij}}, j=1, \ldots, J ; i=1, \ldots, n$,

where, the weight of $w_{i}$ for $i$ th criterion, and $\sum_{i=1}^{n} w_{i}=1$.

\section{E. Step 5. Verify the PIS Distance and NIS Distance}

$A^{*}$ is a positive ideal solutions.

$\left.A^{*}=\mathrm{p}_{1}^{*}, \ldots, \mathrm{p}_{\mathrm{n}}^{*}\right\}=\left\{\left(\max _{j} \mathrm{p}_{\mathrm{ij}} \mid i \in I^{\prime}\right),\left(\min _{j} \mathrm{p}_{\mathrm{ij}} \mid i \in I^{\prime \prime}\right)\right\}$,

$A^{-}$is a negative ideal solutions.

$A^{-}=\left\{\mathrm{p}_{1}, \ldots, \mathrm{p}_{\mathrm{n}}\right\}=\left\{\left(\min _{j} \mathrm{p}_{\mathrm{ij}} \mid i \in I^{\prime}\right),\left(\max _{j} \mathrm{p}_{\mathrm{ij}} \mid i \in I^{\prime \prime}\right)\right\}$,

where $I^{\prime}$ is referred to the positive criteria and $I^{\prime \prime}$ is referred to the negative criteria.

\section{F. Step 6. Design of PIS Distance and NIS Distance}

Euclidean distance is used to calculate the PIS distance

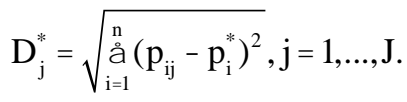
(6).

and Euclidean distance is used to calculate the NIS distance.

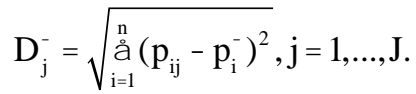

\section{G. Step 7. The Final Calculations of Relative Closeness}

In (11) calculates the best of the alternative $\mathrm{Bi}$ with respect to $\mathrm{S}$,

$$
Q_{j}^{*}=\frac{D_{j}}{D_{j}^{*}+D_{j}}, j=1, \ldots, J .
$$

The most influential alternative is ranked based on (11). The highest value of most influential $Q_{j}^{*}$ refers as the best place over the alternative $\mathrm{Bi}_{\mathrm{i}}$. The highest rank calls as the most effective alternative towards the ideal solution.

\section{RESULTS AND DISCUSSION}

In [24] extent analysis is utilised to calculate the FTOPSIS with affinity weight. This example is used to rank among five recognize cloud solutions for big data projects. Three experts $\left(D_{1}, D_{2}\right.$ and $\left.D_{3}\right)$ are assigned for choosing the most suitable cloud solutions. Five recognize cloud solutions are listed as Amazon as A1, HP Cloud as A2, Google as A3, Rackspace as A4 and Microsoft Azure as A5 versus three criteria: e-Governance as $\mathrm{C} 1$, Business continuity as C2 and Security as C3. The linguistic terms are used by three experts to evaluate the alternatives with respect to different attributes. These linguistic terms Table 1 and 2 are transformed based on the proposed method to sequence computation steps. Thus, this section presents a simplified seven-step computational procedures where the linguistic data are computationally processed to obtain affinity weights of the criteria.

\section{A. Step 1: Linguistic Evaluation and Construct Decision Matrix}

Based on (4) and Table 1, the structure for evaluating the highest cloud solution lead to big data problems is given in Fig. 2 considers all the alternatives and criteria.

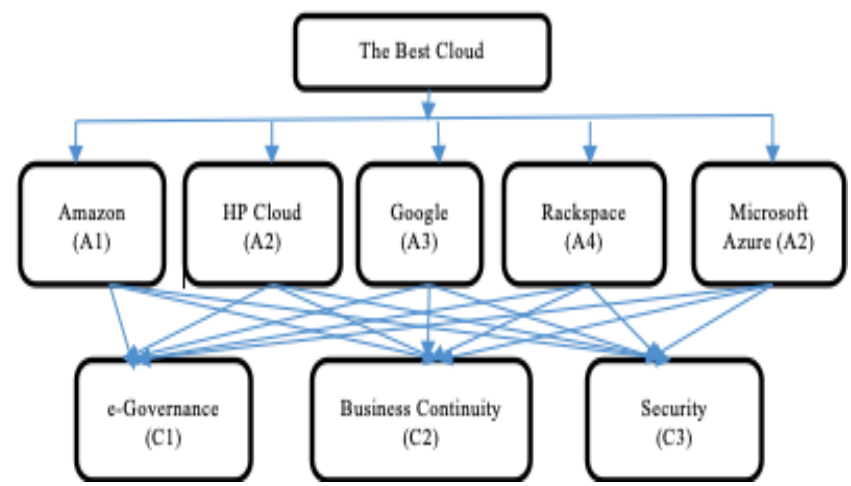

Fig. 2: Hierarchical structure of cloud solution for big data problems 


\section{A FUZZY TOPSIS WITH AFFINITY WEIGHT FOR BIG DATA PROJECTS: MANAGING CLOUD SOLUTION PROBLEMS}

\section{B. Step 2: Normalize the Linguistic Evaluation}

Based on Step 1 and Fig. 2, all values from experts are normalized using (5). This normalizes linguistic evaluations are summarized as Table 3 as follows.

Table 3: Fuzzy decision matrix

\begin{tabular}{|c|c|c|c|c|c|}
\hline & B1 & B2 & B3 & B4 & B5 \\
\hline S & $(0.6333$, & $(0.5$, & $(0.7333$, & $(0.5667$, & $(0.4667$, \\
1 & 0.7333, & 0.6, & 0.8333, & 0.6667, & 0.5667, \\
& $0.8333)$ & $0.7)$ & $0.9333)$ & $0.7667)$ & $0.6667)$ \\
\hline S & $(0.5333$, & $(0.5$, & $(0.6667$, & $(0.4333$, & $(0.3667$, \\
2 & 0.6333, & 0.6, & 0.7667, & 0.5333, & 0.4667, \\
& $0.7333)$ & $0.7)$ & $0.8667)$, & $0.6333)$ & $0.5667)$ \\
\hline S & $(0.6667$, & $(0.5333$, & $(0.6667$, & $(0.6333$, & $(0.5667$, \\
3 & 0.7667, & 0.6333, & 0.7667, & 0.7333, & 0.6667, \\
& $0.8667)$ & $0.7333)$ & $0.8667)$ & $0.8333)$ & $0.7667)$ \\
\hline
\end{tabular}

C. Step 3: Construct the Weighting Using Affinity Weight

Two different hours are selected to manage the big data project for the weighted decision matrix, which are offpeak-hours and peak hours. This is due to the research from [28], there are different type times to access the Internet. Some are on peak hours, some are off-peak hours. Peak hours are during the late evening till midnight time. During this time, the network operators and service providers need to work hard in ensuring its quality and efficiency. Therefore, this study focused on how the e-Governance (C1), Business continuity (C2), Security (C3) towards five market players: Amazon (A1), HP Cloud (A2), Google (A3), Rackspace (A4), Microsoft Azure (A5) during offpeak hours and peak hours.

Linguistic evaluation of affinity weights from Table 2 are used by three experts to evaluate values of the alternatives with respect to attributes towards two different hours; offpeak hours and peak hours respectively. Let's select $\mathrm{C} 1$ as an example to calculate the matrix for off-peak hours is explained as follows.

$$
\mathrm{C} 1:\left(\frac{\mathrm{VI}+\mathrm{VI}+\mathrm{I}}{3}\right)=(7.3333,8.3333,9.3333)
$$

Then, the average is $(0.7333,0.8333,0.9333)$

Table 4: Weight for off-peak hours

\begin{tabular}{|c|c|}
\hline & Weight for Off-Peak Hours \\
\hline S1 & $(0.7333,0.8333,0.9333)$ \\
\hline S2 & $(0.6667,0.7667,0.8667)$ \\
\hline S3 & $(0.4667,0.5667,0.6667)$ \\
\hline
\end{tabular}

Then, the calculation for the matrix of $\mathrm{C} 1$ for off-peak hours is explained as follows.

$$
\mathrm{C} 1:\left(\frac{\mathrm{VI}+\mathrm{VI}+\mathrm{VI}}{3}\right)=(8.0,9 \cdot 0,10)
$$

Then, the average is $(0.8,0.9,1)$

Table 5: Weight or peak-hours

\begin{tabular}{|c|c|}
\hline & Weight for Peak-Hours \\
\hline S1 & $(0.8,0.9,1)$ \\
\hline S2 & $(0.6667,0.7667,0.8667)$ \\
\hline S3 & $(0.5333,0.6333,0.7333)$ \\
\hline
\end{tabular}

\section{Step 4: Form the Decision Matrix's Weighted}

Two decision matrix's weighted for off-peak hours and peak hours are constructed based on Step 2 and 3. Thus, is summarized as Table 6 .

Table 6: Weighted average for off-peak hours

\begin{tabular}{|c|c|c|c|c|c|}
\hline & B1 & B2 & B3 & B4 & B5 \\
\hline S & $(0.4644$, & $(0.3667$, & $(0.5378$, & $(0.4156$, & $(0.3422$, \\
1 & 0.6111, & 0.46, & 0.6944, & 0.5556, & 0.4722, \\
& $0.7778)$ & $0.6067)$, & $0.8711)$ & $0.7156)$ & $0.6222)$ \\
\hline S & $(0.3556$, & $(0.3333$, & $(0.4444$, & $(0.2889$, & $(0.2444$, \\
2 & 0.4856, & 0.46, & 0.5878, & 0.4089, & 0.3578, \\
& $0.6356)$ & $0.6067)$ & $0.7511)$ & $0.5489)$ & $0.4911)$ \\
\hline S & $(0.3111$, & $(0.2489$, & $(0.3111$, & $(0.2956$, & $(0.2644$, \\
3 & 0.4344, & 0.4856, & 0.4344, & 0.4156, & 0.3778, \\
& $0.5778)$ & $0.6356)$ & $0.5778)$ & $0.5556)$ & $0.5111)$ \\
\hline
\end{tabular}

Table 7: Weighted average for peak hours

\begin{tabular}{|c|c|c|c|c|c|}
\hline & B1 & B2 & B3 & B4 & B5 \\
\hline S & $(0.5067$, & $(0.4$, & $(0.5867$, & $(0.4533$, & $(0.3733$, \\
1 & 0.66, & 0.46, & 0.75, & 0.6, & 0.51, \\
& $0.8333)$ & $0.6067)$, & $0.9333)$ & $0.7667)$ & $0.6667)$ \\
\hline S & $(0.3556$, & $(0.3333$, & $(0.4444$, & $(0.2889$, & $(0.2444$, \\
2 & 0.4856, & 0.46, & 0.5878, & 0.4089, & 0.3578, \\
& $0.6356)$ & $0.6067)$ & $0.7511)$, & $0.5489)$ & $0.4911)$ \\
\hline S & $(0.3556$, & $(0.2844$, & $(0.3556$, & $(0.3378$, & $(0.3022$, \\
3 & 0.4856, & 0.4856, & 0.4856, & 0.4644, & 0.4222, \\
& $0.6356)$ & $0.6356)$, & $0.6356)$ & $0.6111)$ & $0.5622)$ \\
\hline
\end{tabular}

E. Step 5: Verify the PIS Distance and NIS Distance

Here, the verification for each PIS distance and the NIS distance are determined as:

$$
\mathrm{A}^{+}=(1,1,1) \mathrm{A}=(0,0,0)
$$

\section{F. Step 6: Design of PIS Distance and NIS Distance}

The Euclidean distance is used to calculate the distance for off-peak hours and peak-hours as stated in Table 8 .

Table 8: The distance off-peak hours

\begin{tabular}{|c|c|c|}
\hline & $\mathrm{D}^{*}$ & $\mathrm{D}^{-}$ \\
\hline B1 & 2.5860 & 2.7550 \\
\hline B2 & 2.8450 & 2.5138 \\
\hline B3 & 2.2903 & 3.0763 \\
\hline B4 & 2.8338 & 2.4937 \\
\hline B5 & 3.1190 & 2.1961 \\
\hline
\end{tabular}

Table 9: The distance peak hours

\begin{tabular}{|c|c|c|}
\hline & $\mathrm{D}^{*}$ & $\mathrm{D}^{-}$ \\
\hline B1 & 2.4250 & 2.9282 \\
\hline B2 & 2.7954 & 2.5400 \\
\hline B3 & 2.1244 & 3.2608 \\
\hline B4 & 2.6816 & 2.6554 \\
\hline B5 & 2.9833 & 2.3389 \\
\hline
\end{tabular}




\section{G. Step 7: The Final Calculations of Relative Closeness}

The comparative results based on closeness coefficients between off-peak hours and peak hours are stated as Table 10 as follows.

Table 10: Closeness coefficients of the proposed method

\begin{tabular}{|c|c|c|c|}
\hline & $\begin{array}{c}\text { Closeness } \\
\text { Coefficients } \\
\text { Off-Peak Hours }\end{array}$ & $\begin{array}{c}\text { Closeness } \\
\text { Coefficients } \\
\text { Peak Hours }\end{array}$ & Results \\
\hline B1 & 0.5158 & 0.5470 & 0.5314 \\
\hline B2 & 0.4691 & 0.4761 & 0.4725 \\
\hline B3 & 0.5732 & 0.6055 & 0.5894 \\
\hline B4 & 0.4681 & 0.4976 & 0.4828 \\
\hline B5 & 0.4132 & 0.4395 & 0.4263 \\
\hline
\end{tabular}

Table 10 shows that B3 is the best cloud solution problems at 0.5894 . The second pace is $\mathrm{B} 1$ at 0.5314 , then B4 at 0.4828, B2 at 0.4725 and lastly B5 at 0.4263 . This ranking can be summarized as $\mathrm{B} 3>\mathrm{B} 1>\mathrm{B} 4>\mathrm{B} 2>\mathrm{B} 5$.

In order to validate this proposed method, we provide a comparative analysis study. Two different comparative analysis using the previous method which are FTOPSIS [14] and Intuitionistic with TOPSIS [24] were used to validate the proposed method. Thus, full results are summarized as Table 11.

Table 11: Comparative analysis

\begin{tabular}{|c|c|}
\hline Methods & $\begin{array}{c}\text { Ranking Order According } \\
\text { to Closeness Coefficient }\end{array}$ \\
\hline Proposed Method & $\mathrm{B} 3>\mathrm{B} 1>\mathrm{B} 4>\mathrm{B} 2>\mathrm{B} 5$. \\
\hline Fuzzy TOPSIS [14] & $\mathrm{B} 3>\mathrm{B} 1>\mathrm{B} 2>\mathrm{B} 4>\mathrm{B} 5$. \\
\hline $\begin{array}{c}\text { Intuitionistic with TOPSIS } \\
{[24]}\end{array}$ & $\mathrm{B} 3>\mathrm{B} 1>\mathrm{B} 4>\mathrm{B} 2>\mathrm{B} 5$. \\
\hline
\end{tabular}

Based on Table 11, we can conclude that all results are well coherent. The slight changes between B4 and B2 maybe due to the inconsistency of each step in different methods.

\section{CONCLUSION}

Performance, schedule, traffic management are three important parts to manage cloud solution problems for big data projects. This paper introduced an affinity set based FTOPSIS method for solving these cloud solution problems. Three committee experts provided weight data using the provided linguistic scales. Based on these three experts, it was found that Google is the best cloud platform compared to Amazon, HP Cloud, Rackspace and Microsoft Azure. Besides, this paper also provided a comparative analysis between the proposed method with two previous methods (FTOPSIS by [14] and Intuitionistic with TOPSIS by [24]). Finally, we can conclude that FTOPSIS with affinity weight is a practical and compatible method to solve big data projects problem. For future work, we aim to integrate affinity sets with other MCDM methods such as AHP or ANP and make a comparison between each of them.

\section{ACKNOWLEDGMENT}

This research was supported by Pusat Pengurusan Kecemerlangan dan Inkubator Penyelidikan, Universiti Sultan Zainal Abidin.

\section{REFERENCES}

1. M. Zeleny, Multiple Criteria Decision Making. New York: McGraw-Hill, 1982.

2. Y. Deng, F. T. S. Chan, Y. Wu, and D. Wang, "A new linguistic MCDM method based on multiple-criterion data fusion," Expert Systems with Applications, 38, 2011, pp. 6985-6993.

3. L. Abdullah, J. Sunadia, and T. A. Imran, "A new analytic hierarchy process in multi-attribute group decision making," International Journal of Soft Computing, 4(5), 2009, pp. 208-2014.

4. R. A. Krohling, and T. T. M. de Souza, "Combining prospect theory and fuzzy numbers to multi-criteria decision making," Expert Systems with Applications, 39(13), 2012, pp. 11487-11493.

5. W. Ho, X. Xu, and P. K. Dey, "Multi-criteria decision making approaches for supplier evaluation and selection A literature review," European Journal of Operational Research, 202(1), 2010, pp. 16-24.

6. E. Hermans, T. Brijs, G. Wets, and K. Vanhoof, "Benchmarking road safety: Lessons to learn from a data envelopment analysis," Accident Analysis and Prevention, 41(1), 2009, pp. 174-182.

7. E. Thanassoulis, M. C. A. S. Portela, and M. Graveney, "Estimating the scope for savings in referrals and drug prescription costs in the general practice units of a UK primary care trust," European Journal of Operational Research, 221(2), 2012, pp. 432-444.

8. S. Boran, and K. Goztepe, "Development of a fuzzy decision support system for commodity acquisition using fuzzy analytic network process," Expert Systems with Applications, 37(3), 2010, pp. 1939-1945.

9. S. Das, and S. Chakraborty, "Selection of non-traditional machining processes using analytic network process," Journal of Manufacturing Systems, 30(1), 2011, pp. 4153.

10. M. Behzadian, S. K. Otaghsara, M. Yazdani, and J. Ignatius, "Review: A state-of the-art survey of TOPSIS applications," Expert Systems with Applications, 39(17), 2012, pp. 13051-13069.

11. T. Özcan, N. Celebi, and Ş Esnaf, "Comparative analysis of multi-criteria decision making methodologies and implementation of a warehouse location selection problem," Expert Systems with Applications, 38, 2011, pp. 9773-9779.

12. C. L. Hwang, and K. S. Yoon, Multiple Attribute Decision Making: Methods and Applications. Berlin: Springer-Verlag, 1981.

13. J-W. Wang, C-H. Cheng, and K-C. Huang, "Fuzzy hierarchical TOPSIS for supplier selection," Applied Soft Computing, 9, 2009, pp. 377-386.

14. C. T. Chen, "Extension of the TOPSIS for group decision-making under fuzzy environment," Journal of Fuzzy Sets and Systems, 114(1), 2000, pp. 1-9.

15. P. U. Onu, X. Quan, L. Xu, J. Orji, and E. Onu, "Evaluation of sustainable acid rain control options utilizing a fuzzy TOPSIS multi-criteria decision analysis model frame work," Journal of Cleaner Production, 141, 2017, pp. 612-625

16. Z. Morteza, F. Mohamad Reza, and M. S. Mortazavi, "Selection of the optimal tourism site using the ANP and fuzzy TOPSIS in the framework if Integrated Coastal Zone Management: A case of Qeshim Island," Ocean and Coastal Management, 130, 2016, pp. 179-187. 
17. K. Rudnik, and D. Kacprzak, "Fuzzy TOPSIS method with ordered fuzzy numbers for flow control in a manufacturing system," Applied Soft Computing, 52, 2017, pp. 1020-1041.

18. A. Hatami-Marbini, and F. Kangi, "An extension of fuzzy TOPSIS for a Group Decision Making with an application to Tehran Stock Exchange," Applied Soft Computing, 52, 2017, pp. 1084-1097.

19. H. T. H. Nguyen, and J. Cao, "Trustworthy answers for top-k queries on uncertain Big Data in decision making," Information Sciences, 318, 2015, pp. 73-90.

20. S. Kaisler, F. Armour, J. A. Espinosa, and W. Money, "Big data: Issues and challenges moving forward," IEEE 46th Hawaii International Conference on System Sciences, 2013, pp. 995-1004.

21. N. Elgendy, and A. Elragal, "Big data analytics: A literature review paper," Industrial Conference on Data Mining, 2014, pp. 214-227.

22. A. Awasthi, and S. S. Chauhan, "A hybrid approach integrating Affinity Diagram, AHP and fuzzy TOPSIS for sustainable city logistics planning," Applied Mathematical Modelling, 36, 2012, pp. 573-584.

23. S. Bag, "Fuzzy VIKOR approach for selection of big data analyst in procurement management," Journal of Transport and Supply Chain Management, 10(1), 2016, pp. 1-6.

24. N. Sachdeva, O. Singh, P. K. Kapor, and D. Galar, "Multi-criteria intuitionistic fuzzy group decision analysis with TOPSIS method for selecting appropriate cloud solution to manage big data projects," International Journal of System Assurance Engineering and Management, 7(3), 2016, pp. 316-324.

25. L. A. Zadeh, "Fuzzy sets," Journal of Information and Control, 8, 1965, pp. 338-353.

26. A. Kaufmann, and M. M. Gupta, Introduction to Fuzzy Arithmetic: Theory and Applications. New York: Van Nostrand Reinhold, 1985.

27. Y-W. Chen, M. Larbani, C-Y., Hsieh, and C-W. Chen, "Introduction of affinity set and its application in datamining example of delayed diagnosis," Expert Systems with Applications, 36(8), 2009, pp. 10883-10889.

28. M. T. Simsim, "Internet usage and user preferences in Saudi Arabia," Journal of King Saud UniversityEngineering Sciences, 23(2), 2011, pp. 101-107. 